\title{
Fecundidade e longevidade de Aphis gossypii Glover, 1877 (Hemiptera, Aphididae) em diferentes temperaturas e cultivares comerciais de crisântemo (Dendranthema grandiflora Tzvelev)
}

\author{
Maria C. de M. Soglia ${ }^{1.2}$ \\ Vanda H. Paes. Bueno ${ }^{1.2}$ \\ Sandra M. M. Rodrigues ${ }^{1}$ \\ Marcus V. Sampaio ${ }^{1}$
}

\begin{abstract}
Fecundity and longevity of Aphis gossypii Glover, 1877 (Hemiptera, Aphididae) at different temperatures and commercial chrysanthemum cultivars (Dendranthema grandiflora Tzvelev). The aphid A. gossypii is one of the main pests in a number of crops both under field and protected conditions. The objective of the present study was to evaluate the fecundity and longevity of $A$. gossypii under different temperatures and commercial chrysanthemum cultivars (Yellow Snowdon, White Reagan and Dark Splendid Reagan) with different trichomes densities $\left(11.3 ; 16.6\right.$ and 21.6 trichome $/ \mathrm{mm}^{2}$ of the leaf, respectively) The trials were carried out in climatic chambers, at four temperatures $\left(15,20,25\right.$ and $\left.30 \pm 1{ }^{\circ} \mathrm{C}\right)$, $70 \pm 10 \% \mathrm{RH}$ and photophase $10 \mathrm{~h}$. The reproductive period significantly decreased with increase of temperature in the three cultivars. In Yellow Snowdon cultivar average duration of the reproductive period was 14.3 days at $25{ }^{\circ} \mathrm{C}$. The maximum fecundity was obtained at the temperature of $25{ }^{\circ} \mathrm{C}$ with 3,$1 ; 2,8$ and 3,6 nymphs/female/day in the Yellow Snowdon, White Reagan and Dark S. Reagan cultivars, respectively. The total fecundity was reduced by extreme temperatures $\left(15\right.$ and $\left.30^{\circ} \mathrm{C}\right)$, and was obtained at $25{ }^{\circ} \mathrm{C}$ with 35,9 nymphs/female. Females maintained in Yellow Snowdon cultivar significantly showed superiority (30,7 nymphs/female) in total fecundity in relation to White Reagan (22,1 nymphs/ female) and Dark S. Reagan (22,9 nymphs/female). The Yellow Snowdon cultivar (with a lower trichome density) had a significant influence in daily and total capacity of nymphs production, showing a higher fecundity of $A$. gossypii females. The aphid's longevity was affected by cultivars and temperature, and this longevity decreased whit increase of temperature. The results showed that there was an interaction between the temperature and host plant on reproductive parameters of $A$. gossypii.
\end{abstract}

KEYWORDs. Aphis gossypii; biology; reproduction; trichome.

\section{INTRODUÇÃO}

Aphis gossypii Glover, 1877 (Hemiptera, Aphididae), apresenta ampla distribuição mundial e encontra-se associado a culturas de grande importância econômica. Constiui-se numa das principais pragas em ambientes protegidos tanto nas regiões de clima temperado como nas de clima tropicais. É uma espécie polífaga, capaz de transmitir mais de 50 viroses de plantas (BLACKMAN \& EASTOP 1984). Esta espécie, assim como Myzus persicae (Sulzer, 1776) é praga primária em cultivos de crisântemo em casas-de-vegetação no Brasil (BUENo 1999).
O aumento da área de produção intensiva da cultura de crisântemo, principalmente no interior de casas-de-vegetação, tem favorecido o aparecimento desses insetos em níveis populacionais capazes de provocar prejuízos econômicos à cultura (BERGMANN et al. 1996). Os pulgões são conhecidos como "estrategistas r", isto é, são muito bem adaptados para explorar temporariamente um novo habitat, através de um rápido aumento da população.

RABASSE (1980) relata que, sob condições de casa-devegetação e na ausência de inimigos naturais, as populações de afídeos são capazes de crescer exponencialmente por um 
período considerável, comumente 0,2 ou 0,3 fêmeas/fêmea por dia. Entretanto, A. gossypii cresce a uma proporção acima de 0,5 fêmeas/fêmea/dia (STEENIS \& El-KhawAss 1995), o que mostra que a população dessa espécie aumenta 33 vezes por semana.

Por outro lado, o potencial reprodutivo dos afídeos pode ser afetado por vários fatores, dentre eles, a qualidade da planta hospedeira, a ocorrência natural de resistência e a temperatura (KocoureK et al. 1994). O estado fisiológico e a morfologia da planta hospedeira determinará a sua resistência inerente, bem como as condições de crescimento da população de afídeos (BETHKE et al. 1998). Dixon (1987) relata, ainda, que uma mudança na temperatura resulta em uma alteração na taxa reprodutiva, assim como na longevidade dos afídeos.

O objetivo desse estudo foi avaliar o efeito de diferentes temperaturas e cultivares comerciais de crisântemo com diferentes densidades de tricomas, sobre alguns parâmetros reprodutivos e a longevidade de $A$. gossypii.

\section{MATERIALE MÉTODOS}

O presente estudo foi conduzido no Laboratório de Biologia do Departamento de Entomologia da Universidade Federal de Lavras, em câmaras climáticas nas temperaturas de 15, 20, 25 e $30 \pm 1{ }^{\circ} \mathrm{C}$, UR de $70 \pm 10 \%$ e fotofase de $10 \mathrm{~h}$. Como substrato de alimentação para os pulgões foram utilizadas folhas de cultivares comerciais de crisântemo de corte Yellow Snowdon, White Reagan e Dark S. Reagan com densidades de 11,3; 16,6 e 21,6 tricomas $/ \mathrm{mm}^{2}$, respectivamente, na superfície abaxial das folhas (Soglia et al. 2002). A metodologia utilizada neste experimento é a mesma usada por SogLiA et al. (2002) uma vez que este estudo é parte complementar do referido artigo.

Folhas das cultivares de crisântemo infestadas com o pulgão A. gossypii, provenientes de colônias de manutenção em casade-vegetação, foram levadas ao laboratório para a obtenção de adultos na fase reprodutiva. Para cada temperatura utilizaramse três recipientes, sendo um para cada cultivar de crisântemo, destinados à criação dos adultos e obtenção de ninfas. Os recipientes consistiram de uma placa de Petri ( $10 \mathrm{~cm}$ de diâmetro) contendo solução estéril de ágar/água a $1 \%$ e uma folha de crisântemo. Em cada folha mantida na placa de Petri foram acondicionadas dez fêmeas adultas ápteras de $A$. gossypii. As placas contendo os insetos adultos foram vistoriadas a cada doze horas para obtenção de ninfas de primeiro ínstar, as quais foram utilizadas para a condução dos estudos biológicos com A. gossypii, nas diferentes cultivares e temperaturas.

Foram utilizadas pequenas câmaras de criação, as quais constituíram as unidades experimentais. Cada unidade foi composta de uma placa de Petri (5 cm de diâmetro) contendo um disco foliar de crisântemo ( $2 \mathrm{~cm}$ de diâmetro), com a face abaxial voltada para cima, fixo no centro da placa sobre $10 \mathrm{ml}$ de solução estéril de ágar/água a $1 \%$ e vedada com filme PVC transparente. Uma ninfa recém-nascida foi transferida para cada unidade experimental, as quais foram acondicionadas em bandejas e mantidas nas respectivas temperaturas. A cada sete dias, as ninfas em desenvolvimento foram transferidas para uma nova unidade experimental contendo um novo disco foliar e submetidas a observações diárias sob microscópio estereoscópico em sala climatizada a $25 \pm 1^{\circ} \mathrm{C}$, até atingirem a fase adulta, quando foram avaliadas a duração do período reprodutivo, fecundidade diária e total de ninfas e a longevidade de A. gossypii.

O experimento foi conduzido em delineamento inteiramente casualizado sob esquema fatorial 3 x 4 (cultivares $\mathrm{x}$ temperaturas) com 10 repetições, sendo cada repetição composta por cinco unidades experimentais. Os resultados referentes ao efeito das cultivares de crisântemo nos parâmetros biológicos do pulgão foram submetidos à análise de variância, seguida do teste de agrupamento de médias de Scott \& Knott ( $\mathrm{P}<0,01$ ou $\mathrm{P}<0,05)$. Para o estudo do efeito da temperatura, a análise de variância foi complementada com análise de regressão e da identidade de modelos (SCOTT \& KNOTT 1974; GomES 1990).

\section{RESULTADOSE DISCUSSÃO}

Período reprodutivo. A temperatura influenciou a duração do período reprodutivo de fêmeas ápteras de $A$. gossypii, decrescendo significativamente com o aumento da temperatura nas três cultivares estudadas (Fig. 1). A duração média desse período, em função das temperaturas e das cultivares estudadas, demonstrou seguir modelo quadrático para a Yellow Snowdon e linear para White Reagan e Dark S. Reagan. Assim, a duração média do período reprodutivo foi de 17,5; 15,2; 14,3 e 3,8 dias para fêmeas mantidas na cultivar Yellow Snowdon, 18,8; 12,0; 10,4 e 2,4 dias para White Reagan e 15,8; 16,2; 9,0 e 3,1 dias na cultivar Dark S. Reagan nas temperaturas de $15,20,25$ e $30^{\circ} \mathrm{C}$, respectivamente (Fig. 1).

Resultados obtidos por Aldyhim \& Khalil (1993) demonstraram que o período reprodutivo de $A$. gossypii foi significativamente menor ( 6,9 dias) a $30^{\circ} \mathrm{C}$, quando comparado às temperaturas de 15,20 e $25^{\circ} \mathrm{C}$ com duração de 22,$1 ; 20,5$ e 20,8 dias, respectivamente. Efeitos adversos de altas temperaturas sobre parâmetros reprodutivos de $A$. gossypii

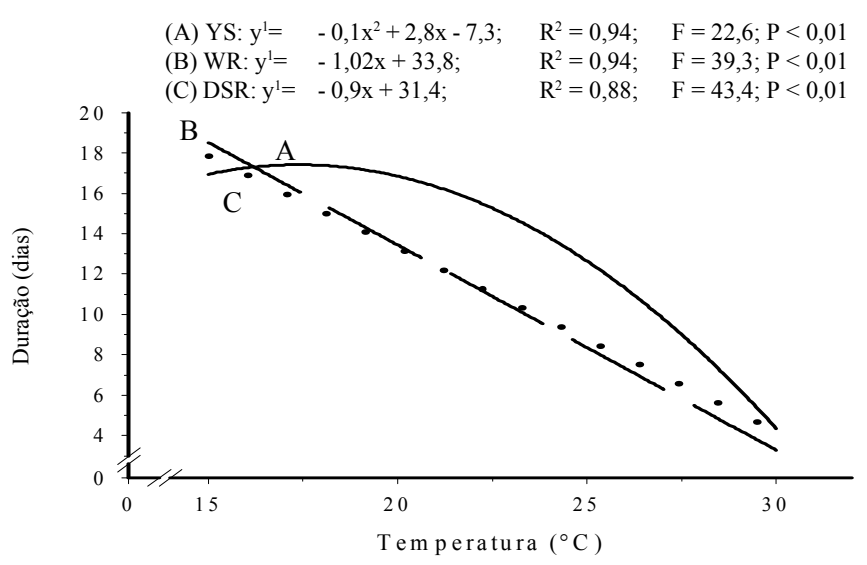

Fig. 1. Duração média do período reprodutivo de fêmeas ápteras de Aphis gossypii em função das temperaturas e cultivares de crisântemo Yellow Snowdon (YS), White Reagan (WR) e Dark S. Reagan (DSR), 70 $\pm 10 \%$ UR, $10 \mathrm{~h}$ de fotofase. 
(A) YS: $\mathrm{y}^{1}=-2,0^{*} 10^{-2} \mathrm{x}^{2}+0,99 \mathrm{x}-8,24 ; \quad \mathrm{R}^{2}=0,90 ; \mathrm{F}=94,72 ; \mathrm{P}<0,01$ (B) WR: $\mathrm{y}^{1}=-2,0^{*} 10^{-2} \mathrm{x}^{2}+1,15 \mathrm{x}-10,48 ; \mathrm{R}^{2}=0,99 ; \mathrm{F}=33,04 ; \mathrm{P}<0,01$ (C) DSR: $\mathrm{y}^{1}=-3,0^{*} 10^{-2} \mathrm{x}^{2}+2,52 \mathrm{x}-14,40 ; \mathrm{R}^{2}=0,83 ; \mathrm{F}=120,58 ; \mathrm{P}<0,01$

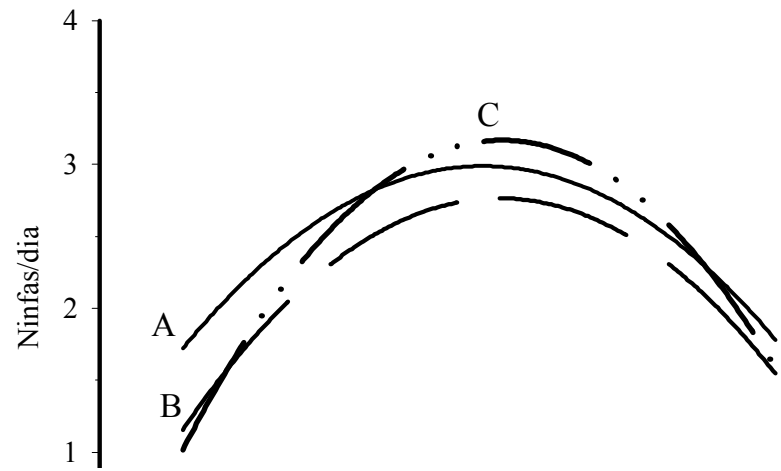

Fig. 2. Fecundidade diária de fêmeas ápteras de Aphis gossypii em função das temperaturas e cultivares de crisântemo Yellow Snowdon (YS), White Reagan (WR) e Dark S. Reagan (DSR), $70 \pm 10 \%$ UR, 10h de fotofase.

foram também reportados por XIA et al. (1999).

Observou-se que apenas na temperatura de $25^{\circ} \mathrm{C}$ houve diferença significativa na duração do período reprodutivo entre as cultivares estudadas, obtendo-se uma maior duração para esse período (14,3 dias), quando fêmeas foram mantidas na Yellow Snowdon (Tabela I). Quando analisada independentemente das temperaturas, a duração média geral obtida para esse período foi de 12,7; 12,0 e 11,2 dias nas cultivares Yellow Snowdon, White Reagan e Dark S. Reagan, respectivamente (Tabela I), demonstrando que as cultivares não afetaram a duração do período reprodutivo de $A$. gossypii nas condições estudadas.

Fecundidade. A fecundidade diária de fêmeas de A. gossypii, em função da temperatura e das três cultivares de crisântemo, seguiu modelo quadrático. Dessa forma, a produção de ninfas/ fêmea/dia na Yellow Snowdon foi de 1,8;2,6;3,1 e 1,7 a 15, 20, 25 e $30^{\circ} \mathrm{C}$, respectivamente (Fig. 2). Na White Reagan e Dark S. Reagan, obteve-se 1,$2 ; 2,4 ; 2,8$ e 1,5 e 1,2; 2,3; 3,6 e 1,4 ninfas/ fêmea/dia, nas temperaturas estudadas, respectivamente (Fig. 2).

A máxima fecundidade diária foi obtida na temperatura de $25^{\circ} \mathrm{C}$ nas três cultivares, enquanto que nos extremos de temperatura, expressos a 15 e $30{ }^{\circ} \mathrm{C}$, ocorreram as menores fecundidades diárias (Fig. 2). O pico de produção diária, obtido na temperatura de $25^{\circ} \mathrm{C}$, foi de 3,1;2,8 e 3,6 ninfas/fêmea/dia nas cultivares Yellow Snowdon, White Reagan e Dark S. Reagan, e a menor produção ocorrendo a $15^{\circ} \mathrm{C}, \operatorname{com} 1,8 ; 1,2 \mathrm{e}$ 1,2 ninfas/fêmea/dia nas respectivas cultivares de crisântemo (Tabela II). Esses valores foram muito próximos aos encontrados por XIA et al. (1999), em algodão para a mesma espécie de pulgão e faixa de temperatura, os quais foram em média 1,2 e 3,1 ninfas, a 15 e $25^{\circ} \mathrm{C}$, respectivamente.

Não houve interação significativa entre as cultivares e as temperaturas testadas para a fecundidade total de $A$. gossypii. Independentemente da cultivar estudada, observou-se que a fecundidade total de fêmeas foi afetada pela temperatura, ajustando-se a um modelo quadrático, segundo o qual a produção máxima de ninfas ocorreu a $25^{\circ} \mathrm{C}$ com 35,9 ninfas (Fig. 3). A $15^{\circ} \mathrm{C}$, a capacidade total de produção de ninfas foi de 24,2 ninfas, valor próximo (21,4 ninfas) ao encontrado por XIA et al. (1999) nessa temperatura. A $20^{\circ} \mathrm{C}$, o número total de ninfas aumentou para 35,7, enquanto que na temperatura de $30^{\circ} \mathrm{C}$, observou-se uma redução significativa na produção total de ninfas com 5,3 ninfas/fêmea (Fig. 3).

De acordo com os resultados, pode-se verificar que a fecundidade de fêmeas partenogenéticas de $A$. gossypii sofreu influência de temperaturas extremas, sendo possível observar que o limite inferior e superior de temperatura, para a menor produção diária e total de ninfas foram estabelecidos a $15 \mathrm{e}$ $30{ }^{\circ} \mathrm{C}$, respectivamente. Esses resultados concordam com observações feitas por EASTOP (1977) em que a temperatura não afeta somente o comportamento individual dos afídeos, mas condiciona o tamanho e a migração das populações de

Tabela I. Duração em dias, (média \pm EP) do período reprodutivo de fêmeas ápteras de Aphis gossypii em função das temperaturas e de cultivares de crisântemo, $70 \pm 10 \% \mathrm{UR}, 10 \mathrm{~h}$ de fotofase.

\begin{tabular}{cccc}
\hline \multirow{2}{*}{ Temperatura $\left({ }^{\circ} \mathrm{C}\right)$} & \multicolumn{3}{c}{ Período Reprodutivo/Cultivares* } \\
\cline { 2 - 4 } & Yellow Snowdon & White Reagan & Dark Splendid Reagan \\
\hline \multirow{2}{*}{15} & $17,5 \pm 0,89 \mathrm{a}(38)$ & $18,8 \pm 1,63 \mathrm{a}(14)$ & $15,8 \pm 1,24 \mathrm{a}(24)$ \\
20 & $15,2 \pm 1,19 \mathrm{a}(33)$ & $12,0 \pm 2,72 \mathrm{a}(11)$ & $16,2 \pm 2,33 \mathrm{a}(15)$ \\
25 & $14,3 \pm 0,72 \mathrm{a}(44)$ & $10,4 \pm 1,43 \mathrm{~b}(17)$ & $9,0 \pm 1,59 \mathrm{~b}(18)$ \\
30 & $3,8 \pm 0,39 \mathrm{a}(35)$ & $2,4 \pm 0,52 \mathrm{a}(08)$ & $3,1 \pm 0,43 \mathrm{a}(12)$ \\
\hline
\end{tabular}

* Médias seguidas pela mesma letra minúscula na linha não diferem estatisticamente entre si pelo teste de Scott \& Knott a 5\% de significância. Valores entre parêntesis expressam o número de observações 
Tabela II. Fecundidade diária de fêmeas ápteras de Aphis gossypii (média \pm EP) em função das temperaturas e de cultivares de crisântemo, $70 \pm$ $10 \% \mathrm{UR}, 10 \mathrm{~h}$ de fotofase.

\begin{tabular}{cccc}
\hline Temperatura $\left({ }^{\circ} \mathrm{C}\right)$ & \multicolumn{3}{c}{ Ninfas/dia/fêmea/Cultivares* } \\
\cline { 2 - 4 } & Yellow Snowdon & White Reagan & Dark Splendid Reagan \\
\cline { 2 - 4 } & $1,8 \pm 0,05 \mathrm{a}(38)$ & $1,2 \pm 0,1 \mathrm{~b}(14)$ & $1,2 \pm 0,09 \mathrm{~b}(24)$ \\
15 & $2,6 \pm 0,14 \mathrm{a}(33)$ & $2,4 \pm 0,43 \mathrm{a}(11)$ & $2,3 \pm 0,19 \mathrm{a}(15)$ \\
20 & $3,1 \pm 0,13 \mathrm{~b}(44)$ & $2,8 \pm 0,18 \mathrm{~b}(17)$ & $3,6 \pm 0,18 \mathrm{a}(18)$ \\
30 & $1,7 \pm 0,09 \mathrm{a}(35)$ & $1,5 \pm 0,19 \mathrm{a}(08)$ & $1,4 \pm 0,19 \mathrm{a}(12)$ \\
\hline
\end{tabular}

*Médias seguidas pela mesma letra minúscula na linha não diferem estatisticamente entre si pelo teste de Scott \& Knott a 5\% de significância. Valores entre parêntesis expressam o número de observações

pulgões.

Embora fêmeas tenham exibido maiores durações do período reprodutivo nas temperaturas de $15 \mathrm{e} 20^{\circ} \mathrm{C}$ (Fig. 1), a temperatura de $25^{\circ} \mathrm{C}$ exerceu maior influência na fecundidade diária e total de fêmeas de $A$. gossypii (Figs. 2 e 3 ).

Em relação ao efeito das cultivares de crisântemo na fecundidade total das fêmeas de $A$. gossypii, observou-se uma diferença significativa dessa característica na Yellow Snowdon em relação às outras, com uma produção total de 30,7 ninfas/ fêmea nessa cultivar. White Reagan e Dark S. Reagan apresentaram uma produção total de 22,1 e 22,9 ninfas/fêmea, respectivamente (Fig. 4).

As menores fecundidades observadas nas cultivares White Reagan e Dark S. Reagan em relação à Yellow Snowdon podem ser explicadas pela maior densidade de tricomas presentes naquelas duas cultivares $\left(16,6\right.$ e 21,6 tricomas $/ \mathrm{mm}^{2}$, respectivamente). A alta densidade de tricomas pode formar uma barreira mecânica, dificultando a penetração dos estiletes de insetos sugadores no tecido das folhas e vasos do floema, podendo provocar menor acúmulo de reservas que,

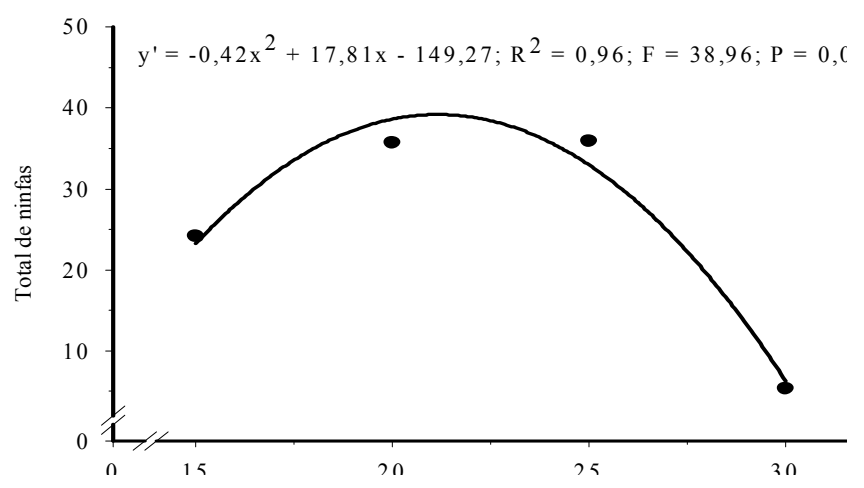

Fig. 3. Fecundidade total de fêmeas ápteras de Aphis gossypii em função da temperatura, $70 \pm 10 \% \mathrm{UR}, 10 \mathrm{~h}$ de fotofase. conseqüentemente, refletem na baixa fecundidade dos adultos. Soglia et al. (2002) relatam que as diferentes densidades de tricomas presentes nas cultivares em estudo, afetou a sobrevivência de ninfas de $1^{\circ}$ e $2^{\circ}$ ínstares de $A$. gossypii. Quando mantidas na cultivar Yellow Snowdon as ninfas apresentaram sobrevivência de $88,5 \%$ e $96,6 \%$ para o $1^{\circ}$ e $2^{\circ}$ ínstares, respectivamente, valores significativamente superiores àqueles obtidos na White Reagan (36,5\% e $80 \%)$ e Dark S. Reagan ( $46 \%$ e $86,3 \%$ ) nesses ínstares. A alta densidade de tricomas presentes nas cultivares impede a locomoção e alimentação dos afídeos acarretando uma baixa sobrevivência.

Estes resultados relacionam-se com afirmações feitas por WyATT (1969), o qual, estudando os fatores que afetam a infestação por afídeos em cultivares de crisântemo, sugeriu que o crescimento da população de afídeos é retardado ou mesmo impedido em cultivares resistentes. Segundo este autor, se plantas susceptíveis e resistentes estiverem em contato, a taxa de crescimento populacional será a mesma em ambas as plantas, porém os números permanecerão proporcionalmente menores sobre a cultivar resistente.

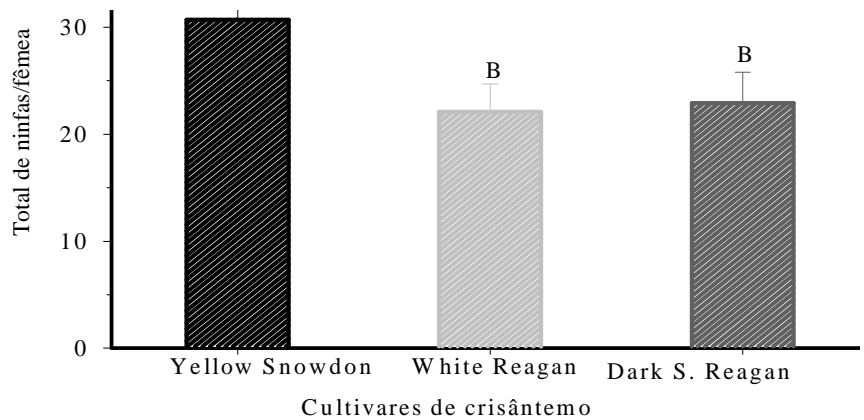

Fig. 4. Fecundidade total de fêmeas ápteras de Aphis gossypii, em função de cultivares de crisântemo. Médias seguidas pela mesma letra não diferem estatisticamente entre si pelo teste de Scott \& Knott a 5\% de significância, $70 \pm 10 \%$ UR, $10 \mathrm{~h}$ de fotofase. 
Tabela III. Longevidade em dias, (média \pm EP) de fêmeas ápteras de Aphis gossypii em função das temperaturas e de cultivares de crisântemo, 70 $\pm 10 \% \mathrm{UR}, 10 \mathrm{~h}$ de fotofase.

\begin{tabular}{cccc}
\hline Temperatura $\left({ }^{\circ} \mathrm{C}\right)$ & \multicolumn{3}{c}{ Longevidade/Cultivares* } \\
\cline { 2 - 4 } & Yellow Snowdon & White Reagan & Dark Splendid Reagan \\
\cline { 2 - 4 } & $27,9 \pm 2,13 \mathrm{~b}(38)$ & $40,8 \pm 4,46 \mathrm{a}(14)$ & $38,5 \pm 3,34 \mathrm{a}(24)$ \\
15 & $21,8 \pm 1,92 \mathrm{a}(33)$ & $14,9 \pm 3,24 \mathrm{~b}(11)$ & $28,2 \pm 5,04 \mathrm{a}(15)$ \\
20 & $18,0 \pm 0,73 \mathrm{a}(44)$ & $12,5 \pm 2,13 \mathrm{~b}(17)$ & $12,0 \pm 1,79 \mathrm{~b}(18)$ \\
25 & $12,5 \pm 1,04 \mathrm{a}(35)$ & $8,7 \pm 2,13 \mathrm{~b}(08)$ & $11,9 \pm 1,39 \mathrm{a}(12)$ \\
30 & &
\end{tabular}

*Médias seguidas pela mesma letra minúscula na linha não diferem estatisticamente entre si pelo teste de Scott \& Knott a 5\% de significância.

Valores entre parêntesis expressam o número de observações

Longevidade. A temperatura exerceu influência significativa na longevidade de fêmeas ápteras de $A$. gossypii. A equação de regressão para a longevidade em função da temperatura e cultivares de crisântemo mostrou-se de natureza linear negativa, decrescendo com o incremento da temperatura. Fêmeas mantidas a $15,20,25$ e $30^{\circ} \mathrm{C}$ apresentaram, na cultivar Yellow Snowdon, uma longevidade média de 27,$9 ; 21,8 ; 18,0$ e 12,5 dias, respectivamente; nas cultivares White Reagan e Dark S. Reagan a longevidade média foi 40,$8 ; 14,9 ; 12,5$ e 8,7 dias e 38,$5 ; 28,2 ; 12,0$ e 11,9 dias, respectivamente a $15,20,25$ e $30^{\circ} \mathrm{C}$ (Fig. 5).

A longevidade de 38,5 dias obtida a $15^{\circ} \mathrm{C}$ para fêmeas de $A$. gossypii mantidas na cultivar Dark $\mathrm{S}$. Reagan, é bem próxima à observada por KeRsting et al. (1999) trabalhando com esta espécie em algodão (39,7 dias). A $20^{\circ} \mathrm{C}$, o valor observado para a longevidade (21,8 dias) na cultivar Yellow Snowdon, aproximase daquele obtido por ALDYHIM \& KHALIL (1993) que, estudando a influência da temperatura no desenvolvimento de $A$. gossypii

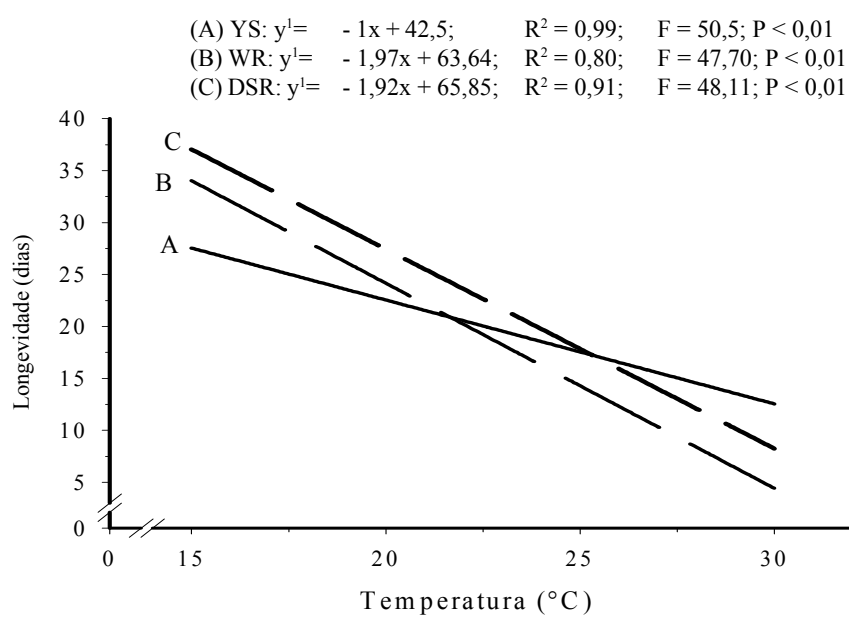

Fig. 5. Longevidade média de fêmeas ápteras de Aphis gossypii em função das quatro temperaturas e cultivares de crisântemo Yellow Snowdon (YS), White Reagan (WR) e Dark S. Reagan (DSR), $70 \pm 10 \%$ UR, 10h de fotofase. sobre plantas de abóbora, constataram uma longevidade média de 25,1 dias nesta temperatura. A 25 e $30^{\circ} \mathrm{C}$, a longevidade média foi significativamente menor, cerca de 18,0 dias para fêmeas mantidas na cultivar Yellow Snowdon e 8,5 para fêmeas mantidas na White Reagan, respectivamente (Tabela III). SteEnIS \& El-Khawass (1995), trabalhando com essa espécie em cultivares de pepino, encontraram uma longevidade média de 14,3 e 14,9 dias para as temperaturas de 25 e $30{ }^{\circ} \mathrm{C}$, respectivamente. Embora se trate de temperaturas iguais às utilizadas nesse estudo, comprova-se pelos valores obtidos que, de certa forma, a longevidade em pulgões é também influenciada diretamente pela planta hospedeira.

É fato conhecido que a temperatura é um fator regulador das atividades dos insetos, e que a qualidade da planta hospedeira e a presença da resistência natural (diferenças entre cultivares) são fatores que interferem no potencial reprodutivo dos afídeos. Assim, a fecundidade de A. gossypii foi afetada pelas temperaturas extremas $\left(15\right.$ e $\left.30^{\circ} \mathrm{C}\right)$ e pelas diferentes cultivares de crisântemo. Fêmeas mantidas a 20 e $25^{\circ} \mathrm{C}$, produziram maior número de ninfas nas cultivares que apresentaram menor densidade de tricomas.

Os resultados obtidos nesse estudo sugerem que há interação entre temperatura e planta hospedeira na biologia de A. gossypii, ocorrendo variações significativas nos parâmetros biológicos avaliados. De forma geral, a cultivar Yellow Snowdon foi a que exerceu maior influência nos parâmetros biológicos avaliados obtendo-se, nessa cultivar, elevada fecundidade, indicando uma maior suscetibilidade à espécie $A$. gossypii em relação às outras cultivares estudadas.

\section{REFERÊNCIAS}

Aldyhim, Y. N.; A. F. KHALIL. 1993. Influence of temperature and daylength on population development of Aphis gossypii on Curcubita pepo. Entomologia Experimentalis et Applicata. 67(2):167-172.

Bergamann, E. C.; S. L. Imenes \& A. P Takematsu. 1996. Pragas. In: S. De L. Imenes \& M. A. V. Alexandre (Coord.). Aspectos Fitossanitários do crisântemo. São Paulo: Instituto Biológico, 41p. (Boletim Técnico, 5).

Bethke, J. A.; R. A. RedaK \& U. K. Schuch. 1998. Melon aphid performance 
on chrysanthemum as mediated by cultivar, and differential levels of fertilization and irrigation. Entomologia Experimentalis et Applicata. 88(1/4):41-47.

Blackman, R. L. \& V. P. EAStop. 1984. Aphids on the world's crops: an identification guide. Chichester: J. Wiley, 466p.

Bueno, V. H. P. 1999. Protected cultivation and research on biological control of pests in greenhouses in Brazil. Integrated control in glasshouses. Bulletin IOBC/WPRS. 22(1): 21-24.

Dixon, A. F. G. 1987. Parthenogenetic reproduction and the rate of increase in aphids. p. 269-287. In: A. K MinKs \& P. HARREWINJN (eds.). World Crop Pest - Aphids: Their biology, natural enemies and control. Amsterdam, Elsevier, v. 2A, 450p.

EAstop, V. F. 1977. Worldwide importance of aphids as virus vectors, p.362. In: K. F Harris \& K. Maramorosch. Aphis as virus vectors. New York: Academic Press 559p.

Gomes, F. P. 1990. O uso da regressão na análise de variância, p.227-243. In: F. P. Gomes (ed). Curso de estatística experimental. São Paulo, Editora Nobel, $13^{\mathrm{a}}$ ed., $468 \mathrm{p}$.

Kersting, U.; S. Satar \& N. Uygun. 1999. Effect of temperature on development rate and fecundity of apterous Aphis gossypii Glover (Homoptera: Aphididae) reared on Gossypium hirsutum L. Journal of Applied Entomology 123: 23-27.

Kocourek, F.; J. Havelka; J. BeránKovÁ \& V. Jarosik. 1994. Effect of temperature on development rate and intrinsic rate of increase of Aphis gossypii reared on greehouse cucumbers. Entomologia Experimentalis et Applicata 71(1): 59-64.

Rabasse, J. M. 1980. Dynamique des populations d'aphides sur aubergine en serre. Considérations générales sur la colonisation et el développement des populations de quatre espéces dans la Sud de la France. IOBC/WPRS, (IOBC/WPRS) Bull. 3(2): 187-198.

Scott, A. J., \& M. A. KNotт. 1974. A cluster analysis method for grouping means in the analysis of variance. Biometrics 30: 507-512.

Soglia, M. C. de M; V. H. P. Bueno \& M. V. Sampaio. 2002. Desenvolvimento e Sobrevivência de Aphis gossypii Glover (Hemiptera: Aphididae) em Diferentes temperatures e Cultivares Comerciais de Crisântemo. Neotropical Entomology 31(2): 211-216.

Steenis, M. J. van \& K. A. M. H. El-Khawass. 1995. Life history of Aphis gossypii on cucumber: Influence of temperature, host plant and parasitism. Entomologia Experimentalis et Applicata 76: 121131.

XIA, J. Y.; W. VAN DeR Werf \& R. Rabbinge. 1999. Influence of temperature on bionomics of cotton aphid, Aphis gossypii, on cotton. Entomologia Experimentalis et Applicata 90: 25-35.

WyATT, I. J. 1969. Factors affecting aphid infestation of Chrysanthemums. Annals of Applied Biology 63(2): 331-337. 